Multilinguisme et créativité littéraire, dir. Olga Anokhina, Louvain-la-Neuve, Academia/ L'Harmattan, coll. " Au cœur des textes, $\mathrm{n}^{\circ} 20$ », 2012, 184 p.

\title{
Sabine Pétillon
}

\section{OpenEdition}

\section{Journals}

Édition électronique

URL : http://journals.openedition.org/genesis/1009

DOI : 10.4000/genesis.1009

ISSN : 2268-1590

Éditeur :

Presses universitaires de Paris Sorbonne (PUPS), Société internationale de génétique artistique littéraire et scientifique (SIGALES)

\section{Édition imprimée}

Date de publication : 15 juin 2013

Pagination : 204-205

ISBN : 978-2-84050-893-9

ISSN : 1167-5101

\section{Référence électronique}

Sabine Pétillon, «Multilinguisme et créativité littéraire, dir. Olga Anokhina, Louvain-la-Neuve, Academia/ L'Harmattan, coll. «Au cœur des textes, n $20 », 2012,184$ p. », Genesis [En ligne], 36 | 2013, mis en ligne le 27 mars 2015, consulté le 22 septembre 2020. URL : http://journals.openedition.org/genesis/ 1009 ; DOI : https://doi.org/10.4000/genesis.1009 
des archives et la valeur génétique du genre épistolaire, qui retrace en même temps les processus d'écriture des poèmes et sert de laboratoire pour leur création. L'archive de Miguel Hernández révèle pour sa part la genèse de l'atelier du poète qui dès l'adolescence et à partir d'opérations comme la réalisation de listes de mots, la copie, la paraphrase, la citation et la traduction s'est donné des outils pour l'exercice de l'écriture.

Bénédicte Vauthier, œuvrant pour une conciliation entre critique textuelle et génétique, expose ses recherches sur l'avanttexte du roman de Juan Goytisolo Paisajes después de la batalla dont elle a préparé une édition critique précédée de préliminaires génétiques (2012). L'étude de plusieurs séquences du roman montre l'appropriation par Goytisolo du pré-écrit ; les transformations effectuées à partir de ses propres écrits dans la presse démontrent la perméabilité et le jeu non seulement entre écriture propre et écriture d'autrui, mais aussi entre l'inédit et le publié. En même temps, les changements entre les différentes éditions du roman permettent d'afficher l'instabilité du texte édité et la porosité des frontières entre avant-texte et texte. Quant à Irène Fenoglio, elle étudie le dossier correspondant à l'incipit de Boutès de Pascal Quignard, composé pour la plupart de tirages d'imprimante des états successifs du récit corrigés à la main par l'auteur. Une place prépondérante dans la genèse de ce « texte-conte » est réservée au dessin du plongeur de Paestum, qui informe l'écrit dès le début. Fenoglio compare ensuite la genèse de cette œuvre de fiction avec celle des manuscrits du linguiste Émile Benveniste.

Enfin, la dernière section est consacrée à l'univers électronique. Jean-Louis Lebrave fait un aperçu historique sur trois acceptions de la génétique électronique : l'électronique comme outil de recherche, l'édition génétique numérique et la composition digitale de textes, ce qui inclut le traitement de texte mais aussi d'autres outils (d'écriture, de recherche, de communication et de disposition d'informa- tion). Le manuscrit électronique est également au centre de l'article de Jesús Rodríguez-Velasco, qui clôt le volume et réoriente la recherche vers l'Internet et l'écriture collaborative. Les deux auteurs s'interrogent sur le déplacement cognitif et épistémologique introduit dans l'acte d'écrire par les ordinateurs et les technologies de l'information. Bien entendu, une des questions que devra approfondir la génétique est de savoir comment ces outils et dispositifs changent notre rapport à l'écriture.

Multilinguisme et créativité langagière, dir. Olga Anokhina, Louvain-la-Neuve, Academia/L'Harmattan, coll. « Au cœur des textes, $n^{\circ} 20 », 2012,184$ p.

\section{Compte rendu par Sabine Pétillon}

Comme le souligne Olga Anokhina dans son propos introductif, le multilinguisme se définit comme la pratique de plusieurs langues à la fois - la configuration la plus usuelle étant celle du bilinguisme qui, lorsqu'il est parfait, permet l'usage d'une langue ou d'une autre indifféremment et surtout sans «mixage » (mélange). Cette excellence dans l'usage de plusieurs langues, les unes étant parfaitement étanches aux autres, est une marque de maîtrise indiscutable. Cependant, il existe des situations où, au contraire, l'usage concomitant de plusieurs langues peut être à l'origine d'une forme extrêmement riche et variée de création.

En effet, et c'est l'objet de ce volume, le mélange de ces langues parfaitement maîtrisées constitue véritablement un médium créatif de premier ordre si l'on observe les manuscrits d'écrivains. Ces manuscrits multilingues permettent aux écrivains d'user d'un système lorsqu'un autre se trouve défaillant : ce que l'un ne permet pas de formuler, un autre le pourra. C'est ainsi que, dans les manuscrits de travail des écrivains, l'on peut observer une langue-support investie, pour ne pas dire envahie, par une autre : au plan du lexique, des constructions grammaticales/ syntaxe - et du style - ainsi, cette interaction linguistique constitue une source inégalable de créativité langagière et littéraire. Et c'est ce que montrent les carnets, les plans, les brouillons : le lecteur s'imagine ne lire qu'une langue alors même qu'il en lit plusieurs : celle qui apparaît sur le papier étant nourrie, imprégnée, influencée par d'autres. On l'aura compris, cet ouvrage nous plonge pleinement dans le vif de la création littéraire et tente d'éclairer - via le multilinguisme - le mystère de l'invention!

L'ouvrage se structure en trois parties. La première évoque les écrivains entre deux langues : entre le russe et le français, le russe et l'anglais, l'italien et le latin traversée par deux langues, l'écriture s'en trouve comme revigorée, démultipliée. Une seconde étape - polyphonique - s'attache notamment à la description de la présence de lexiques étrangers dans le processus créateur. Enfin, la dernière partie de l'ouvrage évoque la question délicate de la traduction et de la cohabitation, au moment même d'écrire, entre plusieurs langues.

C'est avec l'article d'Olga Anokhina que s'ouvre la première partie : « Le rôle du multilinguisme dans l'activité créative de Vladimir Nabokov»(p. 15-25). L'auteur souligne ici que dans les cartes postales ou les notes de Nabokov, le mélange des langues domine (anglais, russe, français), ce qui est moins le cas dans la fiction. Pour ce qui est des traductions, Nabokov traduisait lui-même ses œuvres en russe, mais faisait appel à des traducteurs pour ses œuvres à traduire en anglais. Sur ces dernières traductions, l'écrivain se laissait tout le loisir de retoucher les traductions qu'il considérait finalement comme un premier jet. L'usage de telle ou telle langue peut être orienté par le genre textuel. L'étude de Caroline Béranger, «Écrits français de Marina Tsvetaeva » (p. 27-29), démontre comment la poétesse M. Tsvetaeva a tenté de transposer en langue française une partie des unités lexicales et des constructions syn- 
taxiques propres au russe. Mais le transfert de ces configurations s'est avéré, aux yeux de l'auteur, un échec qui a pu la conduire à cesser cette écriture poétique à deux langues. L'article de Marina Giaveri, « Entre le latin et l'italien : entre la philologie et la critique » (p. 41-54), s'attache à décrire les mécanismes créatifs de Pétrarque (1304-1374), tels que l'on peut les observer à la bibliothèque du Vatican : on peut voir l'écrivain osciller sans cesse entre l'italien et le latin - ce dernier servant notamment aux notes marginales d'autocorrection. Dans son «Étude de manuscrits malgaches bilingues de J.-J. Rabearivelo » (p. 55-65), Claire Riffard montre que l'auteur a choisi de respecter le système linguistique de chaque langue - le français et le malgache - tout en écrivant ses poèmes à la fois dans une langue et dans l'autre. Les deux textes de poèmes évoluant, l'un à côté de l'autre, les structures et le lexique d'une langue pouvaient pénétrer, au cours du processus, l'autre langue, dans un va-et-vient réciproque. Dans « Pratiques et fonctions du multilinguisme dans les journaux russes rédigés en français » (fin XVIII ${ }^{e}$-fin XIXe) (p. 67-82), Catherine Viollet montre comment les jeunes diaristes de l'aristocratie russe se plaisaient à écrire tout aussi bien en français qu'en russe, tout en s'offrant le plaisir régulier de traduire leurs journaux dans d'autres langues encore. Il ne s'agissait donc pas seulement d'écrire, mais de traduire.

La deuxième partie de l'ouvrage s'ouvre sur un article de Valentina Chepiga, «Lexiques d'origine étrangères dans l'œuvre de R. Gary » (p. 83-96). L'auteur nous montre que les manuscrits de Romain Gary, très tôt rédigés en français, sont truffés de mots de langues multiples : russes, anglais, polonais. $\mathrm{Ce}$ mélange des langues implique chez Gary un travail épilinguistique puisqu'il fait suivre les mots étrangers - par exemple les mots russes - d'explications d'ordre sémantique. Sylvie Courtine-Denamy, dans un article intitulé « Plurilinguisme et genèse des textes dans le Journal de pensée de Hannah Arendt » (p. 97-108), nous montre que le journal d'Hannah Arendt (écrit entre 1950 et 1973) implique une véritable gymnastique intellectuelle pour qui voudrait le traduire. Essentiellement écrit dans la langue maternelle de la philosophe, c'est-à-dire en allemand, on trouve un tissage de multiples autres langues : le grec, l'anglais, le latin et le français. Cette pluralité et ce mélange des langues impliquent que l'on puisse se poser la question de l'identité du texte original. L'article suivant, de Daniel Ferrer, «Finnegans Wake ou la créativité multilingue » (p. 109-114), montre que le texte de Finnegans Wake associe de nombreuses langues. L'auteur propose d'évaluer le rôle de ce multilinguisme aux différents stades de la création : des carnets de note aux différents brouillons. Dans «La langue des affects : le cas de Valéry » (p. 110-115), Antonietta Sanna propose d'examiner la pratique de la langue italienne par Valéry, langue maternelle qu'il appelait « langue des affects ». C'est avec cette langue italienne que, dans certains de ses poèmes, de ses titres ou en marge, il parvient à exprimer des expériences sensorielles profondes indicibles pour lui en français.

La dernière partie de l'ouvrage traite de la question du multilinguisme et de la traduction. Dans un premier temps, Chiara Montini évoque «Le rôle du bilinguisme dans la genèse de Mercier and Camier de Samuel Beckett » (p. 129144). Les manuscrits de Mercier and
Camier montrent que Beckett traduit plusieurs fois ses textes avant de les considérer comme matière à réécriture. Il traduit puis retraduit de l'anglais au français, et c'est au terme de ce parcours qu'il efface notamment un certain nombre de gestes affectueux entre les deux protagonistes de la pièce. Dans "L'écriture et l'intraduisible. Le multilinguisme dans la genèse du Précis de décomposition de Cioran » (p. 145-156), $\mathrm{N}$. Cavaillès montre comment le roumain émerge dans les configurations françaises du Précis de décomposition, auquel il convient de joindre d'autres langues comme l'allemand, l'anglais et bien sûr le latin. Ici, l'écriture en français a encore partie liée avec la spontanéité de la langue roumaine. Dans l'ultime article du volume, "L'écriture théorique de Vassily Kandinsky et le problème du multilinguisme » (p. 157-172), Nadia Podzemskaia montre que Kandinsky écrit en trois langues : le russe, l'allemand et le français. Au gré de ses voyages, Kandinsky écrit en russe puis traduit en français, ou écrit en allemand et traduit de nouveau en français ou en russe : il voyage ainsi, non seulement sur un mode géographique, mais sur un mode linguistique, puisant dans chaque langue ce que les autres ne parviennent pas à formuler.

On l'aura compris, cet ouvrage que nous offre Olga Anokhina permet de comprendre quels sont les enjeux du multilinguisme dans la création littéraire, et dans les mécanismes de traductions multiples auxquels s'adonnent un bon nombre d'écrivains : ce que le système d'une langue ne permet pas - induisant un manque dans l'écriture et ce que celle-ci cherche à saisir - une autre langue l'offre et supplée en cela à des formes de creux langagiers inhérents à toute langue. 